\title{
PRÁTICAS DE ALEITAMENTO MATERNO EXCLUSIVO ENTRE PROFISSIONAIS DE SAÚDE DE UM HOSPITAL AMIGO DA CRIANÇA
}

\author{
Rayanne Sousa Melo ${ }^{1}$, Ana Cristina Pereira de Jesus Costa ${ }^{2}$, Leonardo Hunaldo dos Santos ${ }^{3}$, Paula Chuproski \\ Saldan ${ }^{4}$, Marcelino Santos Neto ${ }^{5}$, Floriacy Stabnow Santos ${ }^{6}$
}

\begin{abstract}
RESUMO: O objetivo deste estudo foi identificar a prevalência de aleitamento materno exclusivo entre profissionais de saúde em hospital credenciado como Amigo da Criança e as variáveis de risco para a não adesão ao aleitamento materno exclusivo. Estudo transversal realizado entre janeiro e junho de 2014, com 53 profissionais da saúde que tiveram filhos enquanto trabalhavam em hospital no interior do Maranhão. Dados coletados de instrumento estruturado. Praticaram aleitamento materno exclusivo, até o sexto mês, 15 (28,3\%) profissionais. Facilitou o aleitamento, o alojamento conjunto $(n=45 / 84,9 \%)$, apoio para amamentar por profissionais de saúde, amigos ou familiares $(n=43 / 81,1 \%)$. Dificultaram o aleitamento materno fissuras de mamilo $(n=22 / 41,5 \%)$, dor $(n=20 / 37,7 \%)$, mastite $(n=11 / 20,7 \%)$, ingestão de leites industrializados ( $n=$ nove/ $17 \%)$, água $(n=s e i s / 11,3 \%)$ e chás $(\mathrm{n}=$ quatro/ $7,5 \%)$. O fato de serem profissionais trabalhadoras em hospital "Amigo da Criança" não influenciou de forma positiva no tempo de aleitamento materno exclusivo.
\end{abstract}

DESCRITORES: Aleitamento materno; Prevalência; Pessoal de saúde; Estudos transversais; Hospitais.

\section{EXCLUSIVE BREASTFEEDING PRACTICES AMONG HEALTH PROFESSIONALS OF A BABY FRIENDLY ACCREDITED HOSPITAL}

\begin{abstract}
The present study aimed to identify the prevalence of exclusive breastfeeding among health professionals of a baby friendly accredited hospital and the risk variables for non-adherence to exclusive breastfeeding. Cross-sectional study carried out between January and June 2014, with 53 health professionals who had babies at the time they were working at a hospital in the state of Maranhão. Data was collected with the use of a structured questionnaire. Of these, $15(2.83 \%)$ sustained exclusive breastfeeding for the first six months of their infants' lives. Breastfeeding was facilitated by the following factors: rooming-in facilities $(n=45 / 84.9 \%)$, support to breastfeeding provided by health professionals, friends or relatives $(n=43 / 81.1 \%)$. In turn, breastfeeding was complicated by the following factors: nipple trauma $(n=22 / 41.5 \%)$, pain $(n=20 / 37.7 \%)$, mastitis $(n=11 / 20.7 \%)$, formula milk, babies were given water $(n=\operatorname{six} / 11.3 \%)$ and teas $(n=$ four $/ 7.5 \%)$. The fact that the lactating women performed their activities at a Baby Friendly Accredited Hospital did not have a positive influence on the duration of exclusive breastfeeding.
\end{abstract}

DESCRIPTORS: Breastfeeding; prevalence; Health staff; Cross-sectional studies; Hospitals.

\section{PRÁCTICAS DE LACTANCIA MATERNA EXCLUSIVA ENTRE PROFESIONALES DE SALUD EN HOSPITAL AMIGO DEL NIÑO}

RESUMEN: El objetivo del estudio fue identificar la prevalencia de lactancia materna exclusiva entre profesionales de salud en hospital caracterizado como Amigo del Niño, y variables de riesgo de no adhesión a la lactancia materna exclusiva. Estudio transversal realizado entre enero y junio de 2014, con 53 profesionales de salud que tuvieron hijos mientras trabajaban en hospital del interior de Maranhão. Datos recolectados mediante instrumento estructurado. Practicaron lactancia materna exclusiva, hasta el sexto mes, 15 $(28,3 \%)$ profesionales. Facilitaron la lactancia el alojamiento conjunto $(n=45 / 84,9 \%)$, apoyo para amamantar de profesionales de salud, amigos o familiares ( $n=43 / 81,1 \%)$. Dificultaron la lactancia materna fisuras del pezón $(n=22 / 41,5 \%)$, dolor $(n=20 / 37,7 \%)$, mastitis $(n=11 / 20,7 \%)$, ingesta de leches industrializadas $(n=9 / 17 \%)$, agua $(n=6 / 11,3 \%)$ y tés $(n=4 / 7,5 \%)$. El hecho de tratarse de profesionales trabajadoras en hospital "Amigo del Niño" no influyó positivamente en el tiempo de lactancia materna exclusiva.

DESCRIPTORES: Lactancia Materna; Prevalencia; Personal de Salud; Estudios Transversales; Hospitales.

${ }^{1}$ Enfermeira. Residente do Programa Saúde da Família e Comunidade da Universidade Federal do Tocantins. Palmas, TO, Brasil. ${ }^{2}$ Enfermeira. Doutora em Enfermagem. Docente de Enfermagem da Universidade Federal do Maranhão. Imperatriz, MA, Brasil. ${ }^{3}$ Biólogo. Doutor em Melhoramento Genético. Docente da Universidade Federal do Maranhão. Imperatriz, MA, Brasil.

${ }^{4}$ Nutricionista. Doutora em Ciências. Docente da Universidade Estadual do Centro-Oeste. Guarapuava, PR, Brasil.

${ }^{5}$ Farmacêutico Bioquímico. Doutor em Ciências. Docente de Enfermagem da Universidade Federal do Maranhão. Imperatriz, MA, Brasil.

${ }^{6}$ Enfermeira. Doutora em Ciências. Docente de Enfermagem da Universidade Federal do Maranhão. Imperatriz, MA, Brasil.

Autor Correspondente:

Floriacy Stabnow Santos

Universidade Federal do Maranhão

Av. da Universidade, S/N - 65915-240 - Imperatriz, MA, Brasil

Email: floriacys@gmail.com
Recebido: 06/02/2017

Finalizado: 04/10/2017 


\section{INTRODUÇÃO}

O aleitamento materno (AM) é uma das ações de maior importância para a diminuição das taxas de morbimortalidade infantil e ato insubstituível, visto que o leite materno é o alimento mais completo, em termos de qualidade nutricional, sendo a forma mais segura e barata de se garantir bom estado de saúde para o bebêe $\hat{e}^{(1-3)}$.

Segundo a Organização Mundial da Saúde (OMS), o aleitamento materno exclusivo (AME) deve ser praticado até o sexto mês de vida da criança e complementado com outros alimentos até os dois anos ou mais ${ }^{(4)}$. O AME traz benefícios tanto para a saúde da criança quanto para a da mãe $\mathrm{e}^{(1,3)}$.

Vale ressaltar que a amamentação deve ser abordada e discutida já na primeira consulta de pré-natal realizada pelos profissionais de saúde. Este é o momento ideal para sensibilizar a mãe da importância da amamentação ${ }^{(3,5)}$. Apesar da ampla divulgação e do conhecimento científico sobre a importância do $\mathrm{AME}$, muitas mães não aderem a esta prática ou o fazem parcialmente. Observa-se inclusive que as profissionais de saúde, durante a fase de lactação, podem apresentar dificuldades para a manutenção do AME junto aos seus filhos ${ }^{(1,6)}$.

Diante do impacto positivo do AM para a criança, mãe, família, sociedade e baixa adesão das mães ao AME, o Fundo das Nações Unidas para a Infância (Unicef), juntamente da OMS, lançou em 1991-1992 a Iniciativa Hospital Amigo da Criança (IHAC), que foi incorporada pelo Ministério da Saúde como ação prioritária em 1992. Essa iniciativa tem como finalidade proteger, promover e apoiar a alimentação ideal de lactentes e crianças de primeira infância, com o intuito de conquistar e manter os comportamentos e as práticas necessárias para capacitar mães, famílias e profissionais de saúde a oferecerem a todas as crianças o melhor início de vida ${ }^{(7)}$.

Os profissionais de saúde, sobretudo aqueles pertencentes à IHAC, possuem capacitação quanto ao manejo adequado do AM, o que engloba a importância de disponibilizar à criança AME até o sexto mês de vida. Ressalta-se que, para a certificação da IHAC, é necessário que os hospitais cumpram os Dez Passos para o Sucesso do Aleitamento Materno, o Cuidado Amigo da Mulher e uma série de outros requisitos que buscam a adequada atenção à saúde da mulher e da criança, permitindo, portanto, a hipótese de que as profissionais de saúde que são capacitadas sobre o manejo clínico do $\mathrm{AM}$, adeririam a esta prática nos primeiros seis meses de vida dos filhos ${ }^{(8)}$.

Tendo em vista a importância do AM, a relevância dessa iniciativa que incentiva a amamentação, e a responsabilidade que as instituições carregam consigo por terem o título de IHAC, é primordial observar se as profissionais que trabalham nessas instituições e que foram capacitadas sobre o manejo do AM praticam o AME quando têm seus próprios filhos.

Dessa forma, esta pesquisa objetivou identificar a prevalência de AME entre profissionais de um hospital credenciado como Amigo da Criança, bem como as variáveis de risco para a não adesão ao AME, facilidades e dificuldades encontradas para amamentar.

\section{- MÉTODO}

Trata-se de um estudo transversal, de natureza quantitativa, que mensura ${ }^{(9)}$ a prevalência de AME entre profissionais de saúde, realizado no Hospital Regional Materno Infantil no Estado do Maranhão, Região Nordeste do Brasil, no período de janeiro a junho de 2014. Trata-se da única maternidade pública do sudoeste do Maranhão, credenciada junto a IHAC desde 2001 e, para manter o título, todos os profissionais são rotineiramente treinados para o manejo do $\mathrm{AM}^{(10)}$.

Foram incluídas na pesquisa as profissionais de saúde que faziam parte do quadro de funcionários do HRMI, e que estiveram grávidas e gozaram da licença-maternidade no período compreendido entre janeiro de 2007 e julho de 2013. Foram excluídas as que não tiveram filhos enquanto trabalhavam no HRMI. A partir de informações do Departamento de Recursos Humanos da instituição, 69 funcionárias preenchiam essas condições, sendo que 53 constituíram a amostra desta pesquisa, as 16 restantes não quiseram participar da pesquisa. 
O instrumento de coleta de dados foi um questionário estruturado adaptado ${ }^{(11)}$, preenchido em um único encontro por dois pesquisadores que abordaram as funcionárias em seu local de trabalho. As variáveis pesquisadas foram profissão, escolaridade, idade, estado civil e número de filhos; dificuldades maternas e dos recém-nascidos para amamentação; fatores que facilitaram e dificultaram a amamentação. A prevalência do AME foi calculada dividindo-se o número de crianças que mamaram exclusivamente no seio materno até seis meses pelo número de mulheres estudadas.

Os dados foram digitados e analisados com o auxílio do Microsoft Exce ${ }^{\circledR}$, versão 2016, por meio de estatística descritiva. Foram calculadas frequências absolutas e porcentual dos dados apresentados e, ainda, média e desvio padrão (DP). Para verificar a associação entre as variáveis sociodemográficas e o AME, foram realizados testes não paramétricos de qui quadrado de associação, para testar a correlação entre variáveis categóricas ${ }^{(12)}$. Foram calculadas ainda razões de chance (RC), considerando o intervalo de confiança de $95 \%$. Todos os dados foram tabulados na planilha Excel 2016 e os testes, realizados no programa SAS ${ }^{(13)}$.

A pesquisa foi aprovada pelo Comitê de Ética em Pesquisa da Universidade Federal do Maranhão (parecer 447.459/2013).

\section{RESULTADO}

Das 53 mulheres que participaram da pesquisa, 20 (37,8\%) eram técnicas de enfermagem. A idade oscilou entre 20 e 40 anos, com média de 30,3 anos (DP=4,1). Cursaram Ensino Superior $(n=30 / 56,5 \%)$ e $33(62,3 \%)$ eram casadas. O número de filhos variou de um a três, sendo a média de 1,2 filhos (DP=0,49). Teve seu filho no hospital onde trabalha ( $n=34 / 64,1 \%$ ), o qual tinha IHAC (Tabela 1).

Quanto à prevalência do AM, observou-se que 100\% das mães amamentaram seus filhos, mas, considerando o AME, 15 mulheres (28,3\%) alimentaram exclusivamente seus filhos com leite materno até o sexto mês (Tabela 1).

Tabela 1 - Caracterização das mulheres pesquisadas. Imperatriz, MA, Brasil, 2014 (continua)

\begin{tabular}{lc} 
Características & n (\%) \\
\hline Profissão & \\
\hline Enfermeira & $12(22,6)$ \\
\hline Médica & $6(11,3)$ \\
\hline Técnica em enfermagem & $20(37,7)$ \\
\hline Serviços de apoio & $10(18,9)$ \\
\hline Assistente social & $1(1,9)$ \\
\hline Nutricionista & $4(7,6)$ \\
\hline Situação conjugal & \\
\hline Solteira & $11(20,7)$ \\
\hline Casada & $33(62,3)$ \\
\hline União estável & $9(17)$ \\
\hline Escolaridade & $1(1,9)$ \\
\hline Ensino Fundamental & $22(41,5)$ \\
\hline Ensino Médio & $30(56,6)$ \\
\hline Ensino Superior & \\
\hline Idade, anos & $26(49,1)$ \\
\hline $20-30$ & $27(50,9)$ \\
\hline $31-40$ & \\
\hline Filhos & $44(83,1)$ \\
\hline 1 &
\end{tabular}




\begin{tabular}{lc}
\hline 2 & $7(13,2)$ \\
\hline 3 & $2(3,7)$ \\
\hline Aleitamento materno exclusivo & \\
\hline Sim & $15(28,3)$ \\
\hline Não & $38(71,7)$ \\
\hline Total & $53(100)$
\end{tabular}

Não foi verificada associação entre a prática do AME e as variáveis maternas analisadas (Tabela 2).

Tabela 2 - Características sociodemográficas em relação ao aleitamento materno exclusivo. Imperatriz, MA, Brasil, 2014

\begin{tabular}{|c|c|c|c|c|c|c|}
\hline Variáveis & Categoria & n/total & $\%$ & Valor de $p$ & & RC (IC95\%) \\
\hline \multirow[t]{6}{*}{ Profissão } & Enfermeira & $4 / 12$ & 33,3 & 0,99 & & - \\
\hline & Médica & $2 / 06$ & 33,3 & & & \\
\hline & Técnica em enfermagem & $6 / 20$ & 30 & & & \\
\hline & Serviços de apoio & $3 / 10$ & 30 & & & \\
\hline & Assistente social & $0 * / 1$ & 0 & & & \\
\hline & Nutricionista & $0 * / 4$ & 0 & & & \\
\hline \multirow{3}{*}{$\begin{array}{l}\text { Situação } \\
\text { conjugal }\end{array}$} & Solteira & $4 / 11$ & 36,4 & 0,99 & 2 & $(0,27-14,7)$ \\
\hline & Casada & $9 / 33$ & 27,3 & & 1,31 & $(0,23-7,5)$ \\
\hline & União estável & $2 / 09$ & 22,2 & & Ref & 1 \\
\hline \multirow[t]{3}{*}{ Escolaridade } & Ensino Fundamental & $0 * / 1$ & 0 & 0,92 & & - \\
\hline & Ensino Médio & $8 / 22$ & 36,4 & & & \\
\hline & Ensino Superior & $7 / 30$ & 23,3 & & & \\
\hline \multirow[t]{2}{*}{ Idade (anos) } & $20-30$ & $6 / 26$ & 23,1 & 0,88 & Ref & 1 \\
\hline & $31-40$ & $9 / 27$ & 33,3 & & 1,67 & $(0,18-2,02)$ \\
\hline
\end{tabular}

*Valores zerados impossibilitam o cálculo da razão de chance. RC: razão de chance; IC95\%: intervalo de confiança de 95\%.

Com relação às variáveis de risco para não adesão ao $A M E$, foram observados alguns fatores desmotivadores para a amamentação relacionados tanto com problemas identificados na mulher como no recém-nascido.

Estiveram relacionados às mulheres fissuras de mamilo $(n=22 / 41,5 \%)$, dor ao amamentar $(n=20 /$ $37,7 \%)$, pouco leite $(n=15 / 28,3 \%)$, mastite $(n=11 / 20,7 \%)$, mamilos planos ou invertidos ( $n=\operatorname{seis} / 11,3 \%$ ) e ingurgitamento mamário $(n=u m / 1,9 \%)$. Vale ressaltar que, em alguns casos, a mesma mulher apresentou mais de uma dificuldade para amamentar (Tabela 3). Com relação aos problemas dos recém-nascidos, oito $(30,8 \%)$ crianças tiveram refluxo, $21(39,6 \%)$ usaram chupeta, $36(67,9 \%)$ fizeram uso de bicos artificiais para ingestão de água ou outros líquidos na mamadeira ou chuquinha (Tabela $3)$.

Tabela 3 - Variáveis de risco maternas e dos recém-nascidos para não aderirem ao aleitamento materno exclusivo. Imperatriz, MA, Brasil, 2014 (continua)

\begin{tabular}{ll} 
Variáveis & $\mathbf{n}(\mathbf{\%})$ \\
\hline Maternas & \\
\hline Fissura nos mamilos & $22(41,5)$ \\
\hline
\end{tabular}




\begin{tabular}{lc}
\hline Dor & $20(37,7)$ \\
\hline Pouco leite & $15(28,3)$ \\
\hline Mastite & $11(20,7)$ \\
\hline Mamilos planos ou invertidos & $6(11,3)$ \\
\hline Ingurgitamento mamário & $1(1,9)$ \\
\hline Dos recém-nascidos & $6(23,1)$ \\
\hline Pega incorreta & $2(7,7)$ \\
\hline Bebê que não suga ou sucção fraca & $2(7,7)$ \\
\hline Baixo peso & $2(7,7)$ \\
\hline Hipoglicemia & $3(11,5)$ \\
\hline Intolerância ao leite & $8(30,8)$ \\
\hline Refluxo & $3(11,5)$ \\
\hline Outros & \\
\hline Usou chupeta & $21(39,6)$ \\
\hline Sim & $32(60,4)$ \\
\hline Não & $36(67,9)$ \\
\hline Usou mamadeira ou chuquinha & $17(32,1)$ \\
\hline Sim & $53(100)$ \\
\hline Não
\end{tabular}

Observou-se que quatro $(7,5 \%)$ funcionárias relataram terem voltado ao trabalho com menos de quatro meses, e a maioria $(n=49 / 92,4 \%)$ retornou ao trabalho após quatro meses, mesmo sendo um hospital público com gestão estadual.

Alguns fatores foram considerados facilitadores do AME. Os dados mostram que 41 (77,4\%) crianças foram colocadas para mamar na primeira hora de vida e $45(84,9 \%)$ ficaram com as mães no quarto/apartamento após o nascimento; 43 (81,1\%) mulheres receberam apoio de amigos, familiares ou profissionais de saúde no momento de amamentar (Tabela 4). Alguns fatores dificultaram o AM, como uso de leites industrializados por nove (17\%) crianças logo após o nascimento, seis (11,3\%) que tomaram água e quatro $(7,5 \%)$ que tomaram chás (Tabela 4$)$.

Tabela 4 - Fatores facilitadores e dificultadores para o ao aleitamento materno exclusivo entre as profissionais do hospital. Imperatriz, MA, Brasil, 2014 (continua)

\begin{tabular}{lc}
\hline Fatores facilitadores do aleitamento materno & $\mathbf{n}(\%)$ \\
\hline Mamaram na primeira hora após nascer & \\
\hline Sim & $41(77,4)$ \\
\hline Não & $12(22,6)$ \\
\hline Alojamento conjunto após o nascimento & \\
\hline Sim & $45(84,9)$ \\
\hline Não & $8(15,1)$ \\
\hline Mães apoiadas para amamentar & $43(81,1)$ \\
\hline Sim & $9(17)$ \\
\hline Não & $1(1,9)$ \\
\hline Às vezes & $44(54,3)$ \\
\hline Quem ofereceu apoio & $17(21)$ \\
\hline Familiares & \\
\hline Amigos
\end{tabular}




\begin{tabular}{lc}
\hline Profissionais de saúde & $20(24,7)$ \\
\hline Fatores dificultadores do aleitamento materno & $\mathbf{n}(\%)$ \\
\hline Ingestão de leites industrializados & $9(17)$ \\
\hline Sim & $44(83)$ \\
\hline Não & $6(11,3)$ \\
\hline Ingestão de água & $47(88,7)$ \\
\hline Sim & \\
\hline Não & $4(7,5)$ \\
\hline Ingestão de chá & $48(90,6)$ \\
\hline Sim & $1(1,9)$ \\
\hline Não & $53(100)$ \\
\hline Não lembro
\end{tabular}

\section{- DISCUSSÃO}

Neste estudo, houve análise das práticas de AME entre profissionais de saúde de um hospital, única maternidade pública credenciada à IHAC de toda região e que realiza ações de apoio e promoção do AM tanto entre a clientela interna como externa. Os profissionais de saúde recebem capacitações contínuas sobre o manejo do aleitamento, o que permite estarem sempre atualizados sobre essa temática. A clientela externa, formada em sua maioria pelas mães, recebe informações, incentivo e esclarecimentos sobre a temática em vários momentos, como pré-natal, parto e no pós-parto.

Considerando as profissionais que fizeram parte do estudo, resultados semelhantes foram encontrados em estudos realizados na cidade do Rio de Janeiro (RJ), em uma maternidade pública ${ }^{(11)}$, e em Recife (PE), em uma equipe da Estratégia Saúde da Família ${ }^{(14)}$, na qual as profissionais estudadas tinham perfis sociodemográficos semelhantes às do presente estudo.

Quanto à prática do $\mathrm{AME}, 28,3 \%$ das mulheres amamentaram exclusivamente seus filhos até os seis meses. Estudo realizado em Londrina ${ }^{(15)}$ mostrou que a prevalência do AME em crianças de zero a seis meses foi de $33,8 \%$ e, se apresentado mês a mês, os resultados mostraram prevalências mais altas no primeiro e quarto mês, respectivamente $62,5 \%$ e $47,4 \%$, porém, aos cinco meses, apenas $19,3 \%$ estavam em AME e 7,8\% aos seis meses. Observa-se que, no presente estudo, a prática do AME esteve longe da realidade preconizada pela OMS e pelo Ministério da Saúde, que recomendam o AME até os seis meses $^{(4,16)}$.

Em outro estudo realizado com mulheres de várias profissões, observou-se que a mediana do AME foi de 90 dias (três meses) ${ }^{(17)}$. Constatou-se, portanto, que o fato de a mulher ser profissional de saúde de um hospital credenciado à IHAC não contribuiu para que o AME fosse praticado até o sexto mês, evidenciando que outros fatores, além do conhecimento da técnica de $A M$, são determinantes para o sucesso do AME.

Algumas situações podem representar riscos para a prática do $\mathrm{AME}$, como as fissuras, a dor nos mamilos, o ingurgitamento e a mastite. Estudo transversal realizado em Recife mostrou que 32,1\% das mulheres relataram alguma dificuldade para amamentar ${ }^{(14)}$. Outro estudo quantitativo, realizado no Rio de Janeiro, apresentou resultados semelhantes aos da presente pesquisa, sendo que $34,2 \%$ das mulheres que amamentavam apresentaram fissuras, 32,8\% dor nos mamilos, 11,5\% ingurgitamento, e

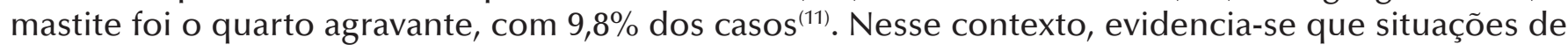
risco, como as encontradas nesta pesquisa, com destaque para fissuras e dor, podem representar um desfecho negativo para a prática do AME.

Identifica-se, no cenário em estudo, que há uma série de problemas relacionados à mulher que afetam diretamente o ato e a decisão de amamentar seu filho, sendo de fundamental importância 
que, desde o pré-natal, a mulher seja orientada sobre a importância do AME, mesmo que ela seja uma profissional de saúde. Alguns destes problemas enfrentados pelas nutrizes durante o AM, se não forem precocemente identificados e tratados, podem ser importantes causas de interrupção da amamentação ${ }^{(14)}$.

Os profissionais de saúde têm um papel importante na prevenção e no manejo dessas dificuldades ${ }^{(3)}$. $\mathrm{O}$ que fazer, entretanto, se elas mesmas apresentam essas dificuldades? Subentende-se que, por serem profissionais de saúde e conhecerem o manejo do AM, teriam discernimento suficiente para agir diante dessas situações sem abandonar o AME até o sexto mês.

No entanto, é preciso refletir sobre isso, pois, mesmo sendo profissionais de saúde de um hospital credenciado como IHAC, essas mulheres são mães e, no momento da amamentação, podem apresentar os mesmos problemas de uma mulher que não é profissional de saúde. Nesse momento, outros fatores, além das técnicas de manejo de AM conhecidas, precisam estar presentes para o sucesso da amamentação, como a rede de suporte dessa mulher, a vontade de amamentar, as condições de saúde da criança e da mulher, e o tempo de permanência com o filho.

O presente estudo mostrou que $41(77,4 \%)$ crianças foram colocadas para mamar na primeira hora de vida. Em outro estudo ${ }^{(18)}$ a prevalência das crianças amamentadas na primeira hora de vida foi de $81,1 \%$, mostrando que ambas as pesquisas estão acima da média nacional, se comparada com II Pesquisa de Prevalência de Aleitamento Materno nas Capitais Brasileiras e Distrito Federal, a qual verificou que, do total de crianças analisadas, 67,7\% mamaram na primeira hora de vida, e em São Luís (MA), onde os índices chegaram a $83,5 \%{ }^{(16)}$.

A atitude de colocar a criança para mamar na primeira hora de vida é um procedimento de suma importância, visto que ele é o quarto passo preconizado nos Dez Passos para o Sucesso do Aleitamento Materno da IHAC ${ }^{(19)}$, mas vale salientar que $36 \%$ das mulheres tiveram seus filhos em hospitais sem IHAC.

Os primeiros dias após o parto são fundamentais para o sucesso da amamentação ${ }^{(3)}$. É importante destacar que, além de colocar a criança no peito logo após a primeira hora de vida, é importante incentivar essa mãe a praticar o alojamento conjunto, sendo este o sétimo passo para o sucesso do AM, segundo o qual as mães e os bebês permanecem juntos 24 horas por dia ${ }^{(19)}$. Estudo mostrou que $62,9 \%$ das mães permaneceram ao lado do filho desde o nascimento até a alta hospitalar ${ }^{(14)}$ mas, no presente estudo, $84,9 \%$ praticaram o alojamento conjunto desde o nascimento, o que indica uma situação favorável e facilitadora do AME.

Apesar da importância do AME, muitas pessoas ainda não estão cientes dos benefícios desse ato. É por essa falta de informação, por insegurança das mães, por elas acreditarem que seu leite seja fraco e pode não suprir as necessidades do filho, muitas introduzem precocemente outros alimentos ${ }^{(7)}$. Estudo apontou que $18,9 \%$ das crianças estudadas usaram leite industrializado, $70 \%$ água e 48,9\% chás, mostrando que tais práticas alimentares eram inadequadas para as crianças ${ }^{(18)}$.

De acordo com a II Pesquisa de Prevalência de Aleitamento Materno nas Capitais Brasileiras e Distrito Federal ${ }^{(16)}$, o consumo de água, chás e outros leites é precoce, acontecendo já no primeiro mês de vida. Na Região Nordeste, 19,1\% das crianças receberam água precocemente e, na Região Sul, esse índice cai para 4,6\%. Quanto ao consumo de leites industrializados, 18\% das crianças os receberam no primeiro mês de vida, com tendência crescente nas faixas etárias subsequentes, chegando a $48,8 \%$ entre 120 e 180 dias. As Regiões Nordeste e Sudeste lideraram a introdução de outros leites no primeiro mês de vida, com cerca de um quinto das crianças já recebendo esse alimento.

O presente estudo mostrou que essa realidade é inferior (17\%) se comparada com esses achados, o que é um ponto positivo. A amamentação é um processo natural e é comum deparar-se com mães em dificuldades durante esse processo, seja por falta de conhecimento de uma pega correta, por falta de tempo ou por insegurança, e por achar seu leite fraco e ineficiente para sustentar a criança ${ }^{(14)}$.

Os profissionais de saúde desempenham importante papel na prática da amamentação, pois estão diretamente em contato com as gestantes desde o pré-natal, realizando orientações e fazendo o acompanhamento necessário, de forma que o sucesso dessas mães no AM é diretamente influenciado por esses profissionais ${ }^{(11)}$. 
É importante destacar que, além da influência dos profissionais de saúde, essas mães sofrem influências da cultura, da família, da comunidade e dos amigos e, para tanto, os profissionais de saúde devem ter habilidade ao exporem às mães as vantagens do AME. Ainda que a mãe seja uma profissional de saúde, ela também está sujeita às mesmas pressões familiares, sociais e emocionais, e, por isso, fazse necessário o aconselhamento profissional que reforce a autoestima e a confiança na capacidade de amamentar ${ }^{(14)}$.

Observou-se que, mesmo com todas essas dificuldades descritas que podem influenciar de forma negativa na amamentação, 67,9\% relataram não ter dificuldade para amamentar, evidenciando o esclarecimento dessas mães sobre o ato de amamentar e percebendo-se que quanto mais essa mulher tem conhecimento do assunto, menos dificuldade ela sente com relação à amamentação, pois ela pode praticar o que aprendeu na teoria.

Estudo realizado no Rio de Janeiro em uma maternidade com IHAC encontrou que a maior dificuldade para o AME foi a pega incorreta $(26,3 \%)^{(11)}$, semelhantemente ao presente estudo, porém também foi relatado pelas profissionais o refluxo como outro dificultador do $\mathrm{AME}^{(11)}$.

Com relação ao uso de chupeta e bicos artificiais das mamadeiras e chuquinhas, seu uso é referido em 38\% e 64,7\% dos casos, respectivamente ${ }^{(14)}$. Dados da II Pesquisa de Prevalência de Aleitamento Materno nas Capitais Brasileiras e Distrito Federal(16) mostraram que, para o total das crianças menores de 12 meses analisadas, foi frequente o uso de mamadeira (58,4\%) e chupeta $(42,6 \%)$.

A mamadeira e os bicos artificiais são considerados fonte de contaminação, favorecendo o aumento dos riscos da mortalidade e morbidade infantil. Além disso, a confusão gerada pela exposição a diferentes técnicas de sucção leva a criança a ter dificuldade de mamar no peito, pois o uso de mamadeiras e chupeta altera a dinâmica oral, promovendo o desmame precoce, proporciona maior risco de preparo de dietas muito diluídas ou concentradas, e reduz o tempo de sucção das mamas, interferindo na amamentação sob livre demanda e retardando o estabelecimento da lactação(7).

O retorno ao trabalho após o término da licença-maternidade também pode representar um problema. Estudo apontou que $94,1 \%$ das mães retornaram ao trabalho aos quatro meses ou mais de vida do bebê $\hat{e}^{(14)}$. O fato de as mães voltarem a trabalhar quando o bebê tinha quatro meses pode ter representado uma dificuldade para manter o AME até os seis meses. A volta ao trabalho no período de 120 dias seria pouco vantajosa com relação à necessidade de AME e aos benefícios que este ato traz.

Foi com o objetivo de prolongar o AM até seis meses que o Governo Federal instituiu o Programa Empresa Cidadã, por meio da lei 11.770, de 9 de setembro de 2008, facultando às empresas a adesão ao programa para prorrogação da licença-maternidade das trabalhadoras em 60 dias, mediante incentivo fiscal. Portanto, o período de 120 dias pode ser estendido para 180 dias, desde que a empregada requeira até o final do primeiro mês após o parto. Dessa forma, a mulher pode se adequar à recomendação do Ministério da Saúde e da OMS de praticar o AME até o sexto mês de vida da criança ${ }^{(20)}$.

Aponta-se como limitação deste estudo a forma de coleta dos dados, que aconteceu em período posterior máximo de sete anos após o parto, o que pode causar um viés de memória.

\section{- CONCLUSÃO}

Ao analisar a prevalência do aleitamento materno exclusivo praticado pelas mulheres, evidenciouse que o fato de serem profissionais que trabalham em um hospital credenciado à Iniciativa Hospital Amigo da Criança não influenciou de forma positiva no aumento do tempo de aleitamento materno exclusivo.

Alguns fatores facilitaram o aleitamento materno exclusivo, como o fato de as mulheres trabalharem em um hospital credenciado à Iniciativa Hospital Amigo da Criança, o que contribui para o esclarecimento que elas têm sobre o manejo do aleitamento materno e sobre a importância desse ato - tanto que todas amamentaram seus filhos.

O fator que dificultou a adesão ao aleitamento materno exclusivo até o sexto mês foi a introdução precoce de líquidos e leites industrializados, o que torna necessário investigar mais a fundo se essa 
decisão foi influenciada por familiares, amigos, ou quais os motivos que as levaram a adotar essa atitude, já que elas mesmas tinham conhecimento sobre os benefícios do aleitamento materno exclusivo até o sexto mês.

Houve variáveis de risco para a não adesão ao aleitamento materno exclusivo, como presença de fissuras no mamilo, dor ao amamentar, mastite, problemas com o próprio recém-nascido, como refluxo (problema fisiológico), e o uso de chupeta e bicos artificiais para ingestão de água e outros líquidos.

Os resultados do estudo contribuem para demonstrar a relevância de mensurar a adesão a práticas de AME entre as profissionais de saúde, a fim de implementar ações de mudança especialmente nas instituições de saúde e garantir essa prática protetiva à saúde infantil, podendo favorecer o ensino e a pesquisa da temática, visto que, no Brasil, diversos estudos discutem o importante papel do AME para a promoção e manutenção da saúde infantil.

\section{AGRADECIMENTOS}

Os autores agradecem o apoio da Fundação de Amparo a Pesquisa e ao Desenvolvimento Científico e Tecnológico do Maranhão - FAPEMA.

\section{- REFERÊNCIAS}

1. Victora CG, Bahl R, Barros AJD, França GVA, Horton S, Krasevec J, et al. Breastfeeding in the 21st century: epidemiology, mechanisms, and lifelong effect. The Lancet. [Internet] 2016;387(10017) [acesso em 28 jun 2016]. Disponível: http://dx.doi.org/10.1016/S0140-6736(15)01024-7.

2. Ministério da Saúde (BR). Secretaria de Atenção à Saúde. Departamento de Atenção Básica. Manual instrutivo das ações de alimentação e nutrição na Rede Cegonha. Brasília (DF): Ministério da Saúde; 2013.

3. Ministério da Saúde (BR). Secretaria de Atenção à Saúde. Departamento de Atenção Básica. Dez passos para uma alimentação saudável. Guia alimentar para crianças menores de dois anos. Um guia para o profissional de saúde da atenção básica. $2^{a}$ ed. Brasília (DF): Ministério da Saúde; 2013.

4. Venancio SI, Saldiva SRDM, Monteiro CA. Secular trends in breastfeeding in Brazil. Rev. Saúde Pública. [Internet] 2013;47(6) [acesso em 04 jul 2015]. Disponível: http://dx.doi.org/10.1590/S0034-8910.2013047004676.

5. da Silva VF, Pessoa CGO. Fatores determinantes do aleitamento materno exclusivo em uma cidade de minas gerais. Revista Enfermagem Integrada. [Internet] 2012;5(1) [acesso em 13 mar 2014]. Disponível: https://www. unilestemg.br/enfermagemintegrada/artigo/v5/01-fatores-determinantes-do-aleitamento-materno-exclusivoem-uma-cidade-de-minas-gerais.pdf.

6. Gomes ARC. Práticas de aleitamento materno em crianças menores de um ano em municípios de Minas Gerais [dissertação]. Belo Horizonte (MG): Universidade Federal de Minas Gerais; 2011.

7. Lopes SS, Laignier MR, Primo CC, Leite FM. Iniciativa Hospital Amigo da Criança: avaliação dos Dez Passos para o Sucesso do Aleitamento Materno. Rev. paul. pediatr. [Internet] 2013;31(4) [acesso em 13 mar 2014]. Disponível: http://dx.doi.org/10.1590/S0103-05822013000400011.

8. Ministério da Saúde (BR). Relação dos Hospitais Amigos da Criança - Brasil - 2014. Brasília (DF): Ministério da Saúde; 2015.

9. Lakatos EM, Marconi MA. Metodologia científica. $6^{\text {a }}$ ed. Atlas; 2011.

10. Fundo das Nações Unidas para a Infância. Iniciativa Hospital Amigo da Criança: revista, atualizada e ampliada para o cuidado integrado: modulo 4: autoavaliação e monitoramento do hospital/ Fundo das Nações Unidas para a Infância, Organização Mundial da Saúde. Brasília: Editora do Ministério da Saúde; 2010.

11. Guimarães LM, da Silva LR, Maques LF. Management of breastfeeding for mothers nursing profesionals, which work in a maternety. J Nurs UFPE on line. [Internet] 2012;6(9) [acesso em 20 jun 2013]. Disponível: http://www. revista.ufpe.br/revistaenfermagem/index.php/revista/article/view/2669/pdf_1420.

http://dx.doi.org/10.5380/ce.v22i4.50523 
12. Callegari-Jacques SM. Bioestatística. Princípios e aplicações. Porto Alegre: Artmed; 2003.

13. Statistical Analysis System (SAS). SAS software: user's guide. Version 8.2. Cary; 2000.

14. Caminha MFC, Serva VB, dos Anjos MMR, Brito RBS, Lins MM, Batista Filho M. Aleitamento materno exclusivo entre profissionais de um Programa Saúde da Família. Ciênc. saúde coletiva. [Internet] 2011;16(4) [acesso em 13 jun 2013]. Disponível: http://dx.doi.org/10.1590/S1413-81232011000400023.

15. de Souza SNDH, Migoto MT, Rosseto EG, de Mello DF. Prevalência de aleitamento materno e fatores associados no município de Londrina-PR. Acta paul. enferm. [Internet] 2012;25(1) [acesso em 12 fev 2014]. Disponível: http:// dx.doi.org/10.1590/S0103-21002012000100006.

16. Ministério da Saúde (BR). II Pesquisa de Prevalência de Aleitamento Materno nas Capitais Brasileiras e Distrito Federal. Brasília (DF): Ministério da Saúde; 2009.

17. Bernardi JLD, Jordão RE, Barros Filho AA. Alimentação complementar de lactentes em uma cidade desenvolvida no contexto de um país desenvolvido. Rev Panam Salud Publica. [Internet] 2009;26(5) [acesso em 15 jan 2014]. Disponível: http://dx.doi.org/10.1590/S1020-49892009001100004.

18. Araújo NL, Lima LHO, Oliveira EAR, Carvalho ES, Duailibe FT, Formiga LMF. Alimentação dos lactentes e fatores relacionados ao aleitamento materno. Rev. Rene. [Internet] 2013;14(6) [acesso em 15 dez 2013]. Disponível: http://www.revistarene.ufc.br/revista/index.php/revista/article/view/1309/pdf.

19. Fundo das Nações Unidas para a Infância (Unicef). Organização Mundial da Saúde (OMS). Iniciativa Hospital Amigo da Criança: revista, atualizada e ampliada para o cuidado integrado. Módulo 4 - Autoavaliação e monitoramento do hospital. Brasília (DF): Ministério da Saúde; 2010.

20. dos Santos KCR, Muraro LO, Witkowski MC, Breigeiron MK. Ganho de peso gestacional e estado nutricional do neonato: um estudo descritivo. Rev. Gaúcha Enferm. [Internet] 2014;35(1) [acesso em 12 fev 2014]. Disponível: http://seer.ufrgs.br/index.php/RevistaGauchadeEnfermagem/article/view/42783. 